Unter dem Vorwand der Rechnungskontrolle wollen Krankenversicherer Zugang zu möglichst vielen Patientendaten, selbstverständlich auch vertraulichen. Dass wirksame abgestufte Kontrollen möglich sind, ohne das für die Behandlung so wichtige Patientengeheimnis zu zerstören, zeigen sowohl die Kodierrevision als auch die Genfer Umsetzung eindrücklich. Zwei Lehrbeispiele, zur Nachahmung empfohlen!

Dr. med. Gert Printzen, Mitglied des Zentralvorstandes

\title{
eHealth und Datenschutz: Wer behandelt die Patienten?
}

Bei eHealth steht der Datenschutz immer mehr zur Disposition. Die Krankenversicherer würden am liebsten das Patientengeheimnis ganz abschaffen. So fordern sie im Zusammenhang mit den elektronischen Patientendaten Zugriff auf das elektronische Patientendossier in gleichem Umfang wie die Ärzte. Und sie fordern die systematische Übermittlung der detaillierten Diagnose- und Prozedurendaten sowie weiterer medizinischer Angaben (Minimal Data Set) mit der Rechnung unter DRG. Deklarierte Zielvorstellung ist, einen «potenziell negativen Krankheitsverlauf frühzeitig zu erkennen und wirksame Massnahmen mit dem Versicherten abzusprechen».[1]. Das bedeutet: Krankenversicherer möchten Kosten senken, indem sie sich in die Behandlung einmischen. Sie sehen sich als die Care Manager - am liebsten wohl analog dem UVG, wo der Unfallversicherer dem Patienten Anordnungen zur Behandlung erteilen kann. Ist das die Rolle, welche die Politik den Krankenversicherern zukommen lassen möchte? Nein. Das Parlament hat beispielsweise die Datenlieferungen unter DRG explizit abgelehnt [2].

BR Couchepin kritisierte die von den Versicherern im Minimal Data Set geforderten Daten als unverhältnismässig: «Wir sind überzeugt, dass die Lösung des Bundesrats und der Mehrheit ausreichend ist, um den Versicherern zu erlauben,

\section{Kontrolle ist möglich, ohne Datenschutz-}

\section{bestimmungen zu verletzen.}

ihre Arbeit zu tun (...) und wir meinen auch, dass man sich im Zweifelsfall eher für den Datenschutz als für die Bequemlichkeit der Versicherer entscheiden muss. Relative Bequemlichkeit, denn sie würden soviele Informationen erhalten, dass sie sie nicht mehr beherrschen könnten.» [3]. NR Yves Guisan ging als Kommissionssprecher noch weiter: «Die Verwaltung dieser beeindruckenden Menge an Daten wird (...) ganz beträchtliche Zusatzkosten generieren. Dies entspricht einer offensichtlich überholten Vorstellung von Kontrollen. (...) Wenn man den Minderheitsvorschlag (...) akzeptiert, dann ist das das Ende des Vertrauensarzt-Systems.» [4].

In der anschliessenden Abstimmung wurde die systematische Übermittlung der relevanten Diagnosen und Prozeduren abgelehnt. Was ist seither geschehen?

In Deutschland belaufen sich die Administrativ-Kosten für die DRG-Überprüfung auf 350 Euro pro Fall. In der
Schweiz wirbt so manch ein Versicherer den Spitälern die Kodierer ab und baut eigene Abteilungen auf. Die vertrauensärztlichen Dienste müssten in Zukunft stark ausgebaut werden, ebenso wie die Informatik-Infrastruktur bei den Versicherern.

Die Versicherer verfügen seit mehr als einem Jahrzehnt über die Daten des Minimal Data Sets in anonymisierter Form. Doch haben sie sich bis heute nicht die Mühe gemacht, aufzuzeigen, wie sie diese Daten verwenden wollen, geschweige denn aufgezeigt, wofür sie sie benötigen. WZWKriterien scheinen demnach nicht für Versicherer zu gelten.

Die Kodier-Revision durch externe Dritte stellt das einzige wirkungsvolle Instrument zur Kodierkontrolle dar. Es ist in der Schweiz etabliert.

Das Universitätsspital Genf und die Versicherer haben ein Verfahren etabliert, das den Geboten des Datenschutzes und der heutigen Gesetzeslage entspricht: Dort werden die kodierten Diagnosen und Prozeduren sowie die wichtigsten Berichte zu einem Spitalaufenthalt nur auf Anforderung durch die Versicherung und ausschliesslich im Einzelfall an den Vertrauensarzt geliefert. Nur bei ca. 20-30 von über 43000 Rechnungen pro Jahr kommt es aufgrund einer korrigierten Kodierung zu einer Korrektur der Rechnung. Im Übrigen ging die Zahl der Fälle, bei denen die Unterlagen angefordert wurden, innerhalb von 2 Jahren um $20 \%$ zurück. Trotzdem werden die Kassen nicht müde, die Daten weiterhin zu verlangen. Und nun scheint das BAG ausgerechnet das, was das Parlament explizit abgelehnt hat, über Tarifverträge umsetzen zu wollen.

Dabei zeigen sowohl die Kodierrevision wie auch das Genfer Beispiel eindrücklich, dass es durchaus möglich ist, wirksame abgestufte Kontrollen durchzuführen und dabei das für die Behandlung so wichtige Patientengeheimnis zu wahren.

Dr. sc. hum. Judith Wagner, Leiterin eHealth FMH Hanspeter Kuhn, Fürsprecher, stv. Generalsekretär FMH

1 Zellweger U. Helsana AG. 8.2. Leistungsabwicklung in der Krankenversicherung - automatisierte Verarbeitung von Leistungen. In: Oggier W et al. Gesundheitswesen Schweiz im Umbruch.

2 Vgl. Kuhn HP. Urteil vom 29. Mai 2009: kein Leitentscheid für die Einführung von SwissDRG. Schweiz Ärztezeitung. 2011;92(16/17):605

3 AB 2007 N 444f; Übersetzung FMH.

4 AB 2007 N 445; Übersetzung FMH. 